\title{
Ballistic two-dimensional lateral heterojunction bipolar transistor
}

\author{
Leonardo Lucchesi $\odot^{*}$ \\ Department of Physics “Enrico Fermi,” University of Pisa, 56127 Pisa, Italy \\ and Department of Information Engineering, University of Pisa, 56122 Pisa, Italy \\ Gaetano Calogero, Gianluca Fiori, and Giuseppe Iannaccone $\odot^{\dagger}$ \\ Department of Information Engineering, University of Pisa, 56122 Pisa, Italy
}

(Received 2 December 2020; accepted 24 March 2021; published 28 May 2021)

\begin{abstract}
We propose and investigate the intrinsically thinnest transistor concept: a monolayer ballistic heterojunction bipolar transistor based on a lateral heterostructure of transition metal dichalcogenides. The device is intrinsically thinner than a field effect transistor because it does not need a top or bottom gate, since transport is controlled by the electrochemical potential of the base electrode. As is typical of bipolar transistors, the collector current undergoes a tenfold increase for each $60 \mathrm{mV}$ increase of the base voltage over several orders of magnitude at room temperature, without sophisticated optimization of the electrostatics. We present a detailed investigation based on self-consistent simulations of electrostatics and quantum transport for both electrons and holes of a $p$ - $n$ - $p$ device using $\mathrm{MoS}_{2}$ for the 10-nm base and $\mathrm{WSe}_{2}$ for the emitter and collector. Our three-terminal device simulations confirm the working principle and a large current modulation $I_{\mathrm{ON}} / I_{\mathrm{OFF}} \sim 10^{8}$ for $\Delta V_{\mathrm{EB}}=0.5 \mathrm{~V}$. Assuming ballistic transport, we are able to achieve a current gain $\beta \sim 10^{4}$ over several orders of magnitude of collector current and a cutoff frequency up to the THz range. The exploration of the rich world of bipolar nanoscale device concepts in two-dimensional materials is promising for their potential applications in electronics and optoelectronics.
\end{abstract}

DOI: 10.1103/PhysRevResearch.3.023158

\section{INTRODUCTION}

The bipolar junction transistor (BJT) has been the first semiconductor transistor manufactured in volume [1] and for 30 years the workhorse of semiconductor electronics, before being taken over by the metal-oxide-semiconductor field-effect transistor (MOSFET). Still, as of today, the heterojunction bipolar transistor (HBT) is the fastest transistor [2], and is the device of use in applications where high power and very high frequency are required, such as telecommunication stations and satellite communications. In addition, BJTs are largely used in ubiquitous building blocks of integrated circuits, such as band-gap voltage references and temperature sensors.

Indeed, device physicists and engineers are much more familiar with MOSFETs than BJTs, and the recent explosion of interest for electron devices based on two-dimensional (2D) materials has been mainly focused on MOSFETs [3,4], for the possibility of enabling an extension, or even an acceleration, of the so-called Moore's law, i.e., the exponential increase of the number of transistors in an integrated circuit as a function

\footnotetext{
*leonardo.lucchesi1@phd.unipi.it

$\dagger$ giuseppe.iannaccone@unipi.it

Published by the American Physical Society under the terms of the Creative Commons Attribution 4.0 International license. Further distribution of this work must maintain attribution to the author(s) and the published article's title, journal citation, and DOI.
}

of time. This possibility is predicated on the fact that $2 \mathrm{D}$ materials can provide an extremely thin layer with a relatively high mobility, thereby enabling scaling of the channel width and length while preserving a good electrostatic behavior and low delay times.

While BJTs and MOSFETs are very similar devices in terms of potential-barrier-controlled transport, BJTs have the advantage of the collector current increasing by a factor 10 for every $60 \mathrm{mV}$ of increase of the base voltage for several decades, at room temperature, up to very high current density. The drain current of a MOSFET has a similar exponential dependence on the gate voltage only at very low current, in the so-called "subthreshold region," and shows a slower exponential. The reason for this difference is that in both devices the main current is controlled by modulating the barrier between the central region (the base for the BJT and the channel for the MOSFET) and the emitting electrode (the "emitter" in the BJT and the "source" in the MOSFET). The advantage of the BJT, in this case, is simply that the base region is in direct contact with a metal electrode, therefore a change of the voltage applied to the base electrode is directly transferred to the electrostatic potential in the base, whereas in the case of the MOSFET the channel region is only capacitively coupled to the gate electrode through the gate dielectric layer, and there is a voltage partition between the potential on the gate and the potential in the channel. We highlight the negligible gate current as the big advantage of the MOSFETs, whereas the BJT has a non-negligible base current, that is a factor $\beta$ smaller than the collector current (where the current gain $\beta$ is in the range $10-10^{3}$ ), and that typically increases the power 

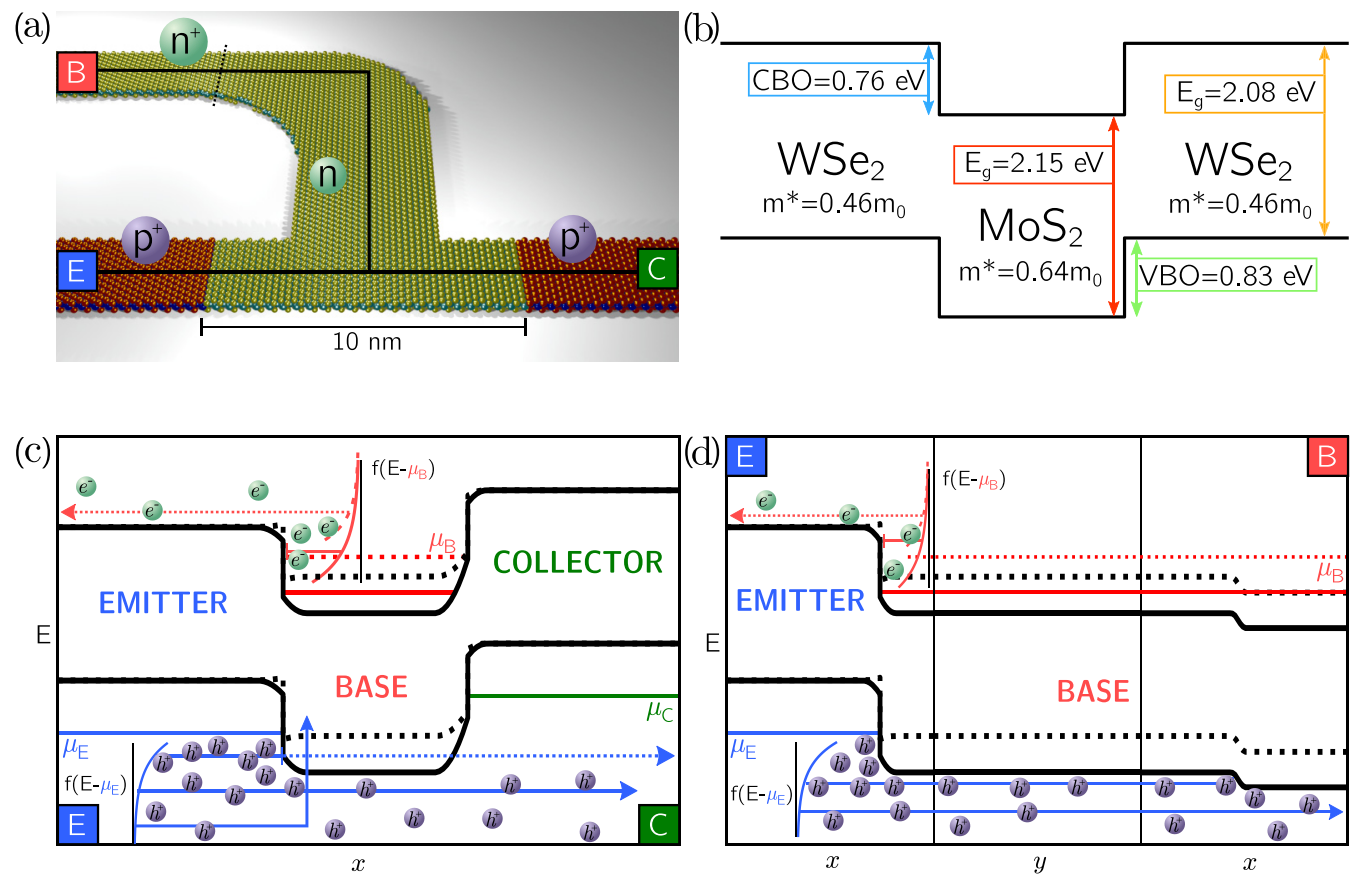

FIG. 1. Device layout and working principle. (a) Artistic illustration of the device. We indicate materials doping and the emitter-collector (EC) and emitter-base (EB) paths along which we show most physical quantities. Leads are represented with letters: emitter (E), base (B), and collector (C). (b) Band alignment of materials (see Appendix A). The working principle is represented in (c) for the EC path, and in (d) for the EB path. Black lines are band edges (solid $\mu_{\mathrm{B}}=1.90 \mathrm{eV}$, dotted $\mu_{\mathrm{B}}=2.38 \mathrm{eV}$ ). Blue/green lines are emitter/collector chemical potentials $\left(\mu_{\mathrm{E}}=0.0 \mathrm{eV}, \mu_{\mathrm{C}}=0.5 \mathrm{eV}\right)$, and red solid/dotted lines are the base chemical potential for $\mu_{\mathrm{B}}=1.90 / 2.38 \mathrm{eV}$. Arrows and spheres represent carrier flows, and the upward arrow in the middle plot is the emitter to base hole flow. For $\mu_{\mathrm{B}}=1.90 \mathrm{eV}$, the base barrier blocks hole flow from $\mathrm{E}$ to $\mathrm{B}$ (OFF state). When $\mu_{\mathrm{B}}$ is raised to $2.38 \mathrm{eV}$, the barrier rises, and more holes flow (ON state). However, the electron flow from $\mathrm{B}$ to $\mathrm{E}$ (dotted red arrow in both plots) and hole flow from $\mathrm{E}$ to $\mathrm{B}$ are also increased. A more detailed discussion can be found in the text.

consumption of circuits based on BJTs. At the nanoscale and at very low current density, BJTs can again be promising as compared to MOSFETs, because a subthreshold swing close to $60 \mathrm{mV} /$ decade in BJTs is obtainable without the use of extremely thin dielectric layers and sophisticated electrostatics engineering, and because source-to-drain leakage currents can become comparable to base currents, if $\beta$ is sufficiently high.

Among the classes of 2D materials, transition metal dichalcogenides (TMDCs) are one of the most promising for transistor use [5-8], offering a wide range of properties and supporting relatively inexpensive, stable and scalable fabrication techniques. Among those, one-step chemical vapor deposition (CVD) growth is establishing a quality standard for devices $[8,9]$, but the realization of TMDC lateral $p-n$ junctions is still a complex issue [10]. Two contributions in this direction stand out: a two-step CVD growth by Li et al. [10] that allows the growth of atomically thin $\mathrm{WSe}_{2}-\mathrm{MoS}_{2}$ $p$ - $n$ junctions, and a morphological method by Han et al. [11] for growing narrow $\mathrm{MoS}_{2}$ channels embedded in $\mathrm{WSe}_{2}$. The type-II heterostructure shown in Fig. 1(b) is obtained in both cases, making these methods suitable for the production of heterostructure bipolar junction transistors (HJTs) as demonstrated by Lin et al. [12].

In this paper, we propose the concept of a ballistic lateral heterojunction bipolar transistor based on transition metal dichalcogenides (TMDCs), and assess its potential in electronics applications with self-consistent quantum transport and electrostatics simulations using the nonequilibrium
Green's function (NEGF) formalism [13,14]. We highlight that, despite the fact that BJTs have received little attention by the vibrant global $2 \mathrm{D}$ materials research community, some experimental demonstrations have recently appeared in the literature, based both on lateral $[12,15]$ and vertical $[16,17]$ operations. The main challenges in the fabrication of 2D BJTs reside in effectively doping the regions and in obtaining highquality heterojunctions. From a computational point of view, the main challenge is the modeling of two-carrier flows in a far-from-equilibrium multilead setup, that is a notoriously challenging problem and has been added as a capability to the NANOTCAD VIDES package [18], the software used for all simulations in this work.

\section{METHODS}

\section{Model}

In order to describe our system, we use a nearest-neighbor effective mass tight-binding model [19]. In this model, materials are described by a tight-binding Hamiltonian whose band structure has the same band gap, electron affinity, and band curvature (effective mass) as the original material. Transport is assumed to be completely ballistic (see Appendix B). The system is solved by using an NEGF-Poisson iterative self-consistent procedure, where we use NEGF to compute currents between leads and the charge ballistically injected from the leads into the device region, solve a Poisson problem for that charge, and use the resulting potential as an input 

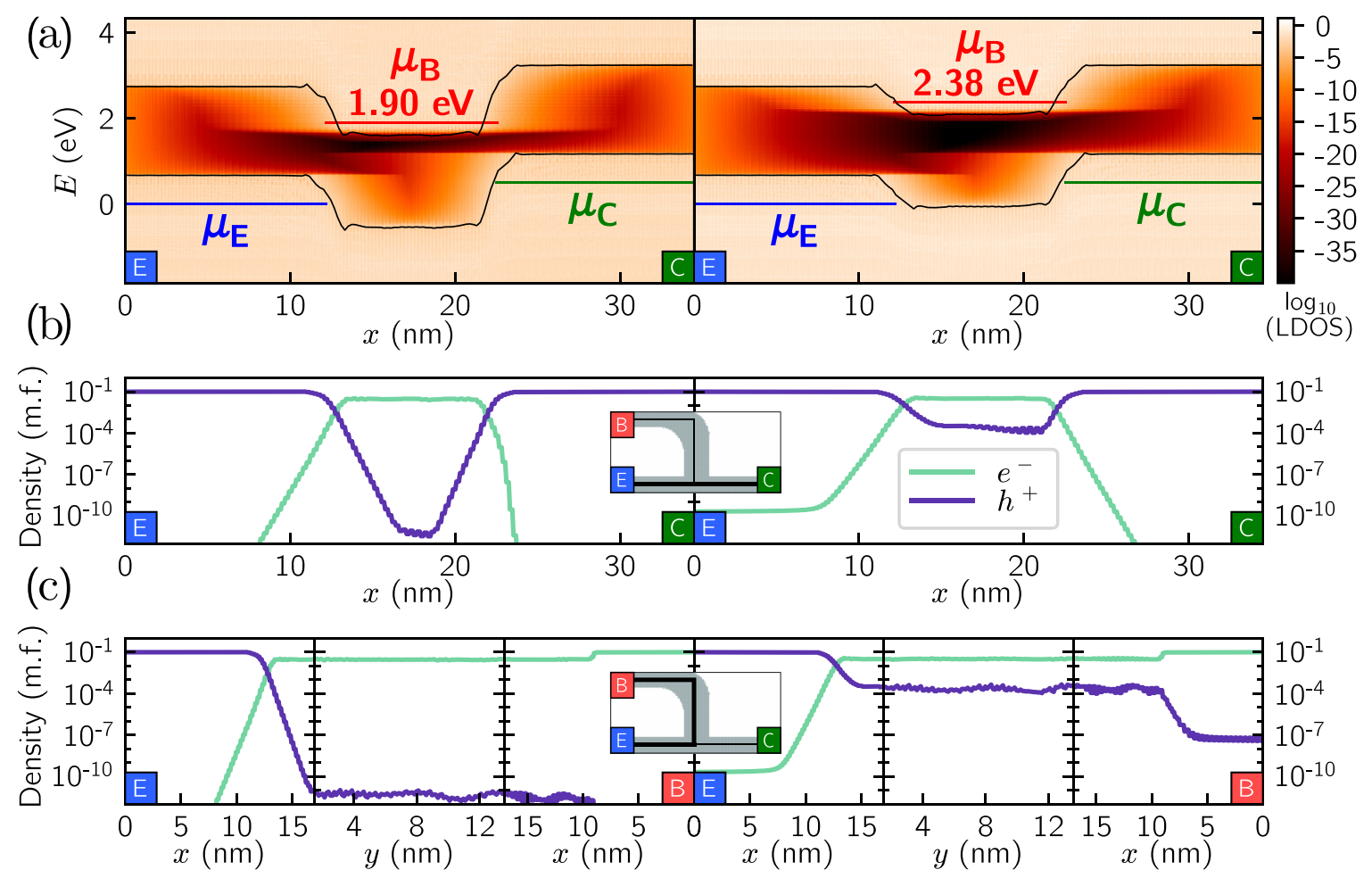

FIG. 2. Demonstration of working principle. (a) Local density of states (LDOS) along the EC path for $\mu_{\mathrm{B}}=1.90 / 2.38 \mathrm{eV}$. Black lines represent band edges, and colored straight lines are lead chemical potentials. Change in LDOS following change $\Delta \mu_{\mathrm{B}}$ confirms the working principle: The main difference is a $\Delta \mu_{\mathrm{B}}$ rigid shift in the bands (no gate present). (b) and (c) Injected carrier concentrations (molar fraction) respectively for the EC path and EB path, both shown for $\mu_{\mathrm{B}}=1.90 / 2.38 \mathrm{eV}$. Insets are path references. Charge neutrality is confirmed in the whole device, the central region included (nontrivial), as the injected carrier concentration is equal and opposite to doping. Concentrations lower than $10^{-12}$ are neglectable.

to the next NEGF step. The simulation of device operation requires proper handling of two main issues: the presence of three leads in a far-from-equilibrium condition common in real nanoscale devices and the need for a self-consistent description of the transport and electrostatics. As we are considering TMDCs, we can use a hexagonal lattice with a lattice parameter $d$ and two effective atoms in the primitive cell, each with a single energy level. The Hamiltonian reads

$$
\left[\begin{array}{cc}
E_{0} & t f(\vec{k}) \\
t f(\vec{k}) & E_{1}
\end{array}\right]
$$

where $f(\vec{k})=\left(1+e^{-i \vec{k} \cdot \vec{a}}\right)$. The bands generated by this Hamiltonian have a direct gap $E_{\text {gap }}=E_{0}-E_{1}$ in the $K$ point. Close to $K$, the dispersion relation can be described as free, with an effective mass that is related to the hopping parameter $t$ by

$$
m^{*}=\frac{\hbar^{2}}{q} \frac{2 \Delta}{9 d^{2}|t|^{2}},
$$

where $d$ is the interatomic distance and $q$ is the electron charge. In our nearest-neighbor model, the effective mass results to be the same for the valence and the conduction band, restricting the validity of this Hamiltonian to those materials whose electron and hole effective masses are similar. Both $\mathrm{WSe}_{2}$ and $\mathrm{MoS}_{2}$ fall into this category (see Appendix A). Heterojunctions have been described via a linear interpolation of all Hamiltonian parameters in their proximity.

\section{RESULTS}

\section{A. Working principle}

We consider a $p-n-p$ double heterojunction bipolar transistor (DHBT) with 2D materials, with an $\mathrm{MoS}_{2}$ base, and $\mathrm{WSe}_{2}$ emitter and collector. We consider a nanoribbon channel and the possibility of heavily doping the $2 \mathrm{D}$ regions, so that the device structure under investigation is illustrated in Fig. 1(a). Let us stress that transistors work correctly even if there is no lateral confinement, and that effective doping is required for a device practically usable in circuits. Therefore, in order to assess the potential of $2 \mathrm{D}$ BJTs, we need to assume that technological challenges in the doping of $2 \mathrm{D}$ materials on a large scale can be solved, as has been demonstrated in laboratory conditions $[5,6]$.

The device operation is represented in Figs. 1(c) and 1(d) through ideal band edge profiles, respectively along the emitter-to-collector path and along the emitter-to-base path. A thermionic hole current flowing between the emitter and collector $\mathrm{WSe}_{2}$ regions is controlled by modulating a barrier in the central $\mathrm{MoS}_{2}$ region (base). This barrier is modulated by changing the electrochemical potential of the base lead, i.e., injecting electrons in the base region. This modulation causes a shift in the electrostatic potential in the base through the unbalance between the charge injected from the leads and the fixed charge due to materials doping. We can find an easy interpretation for this shift by inspecting the bulk of each region, i.e., more than a screening length away from 

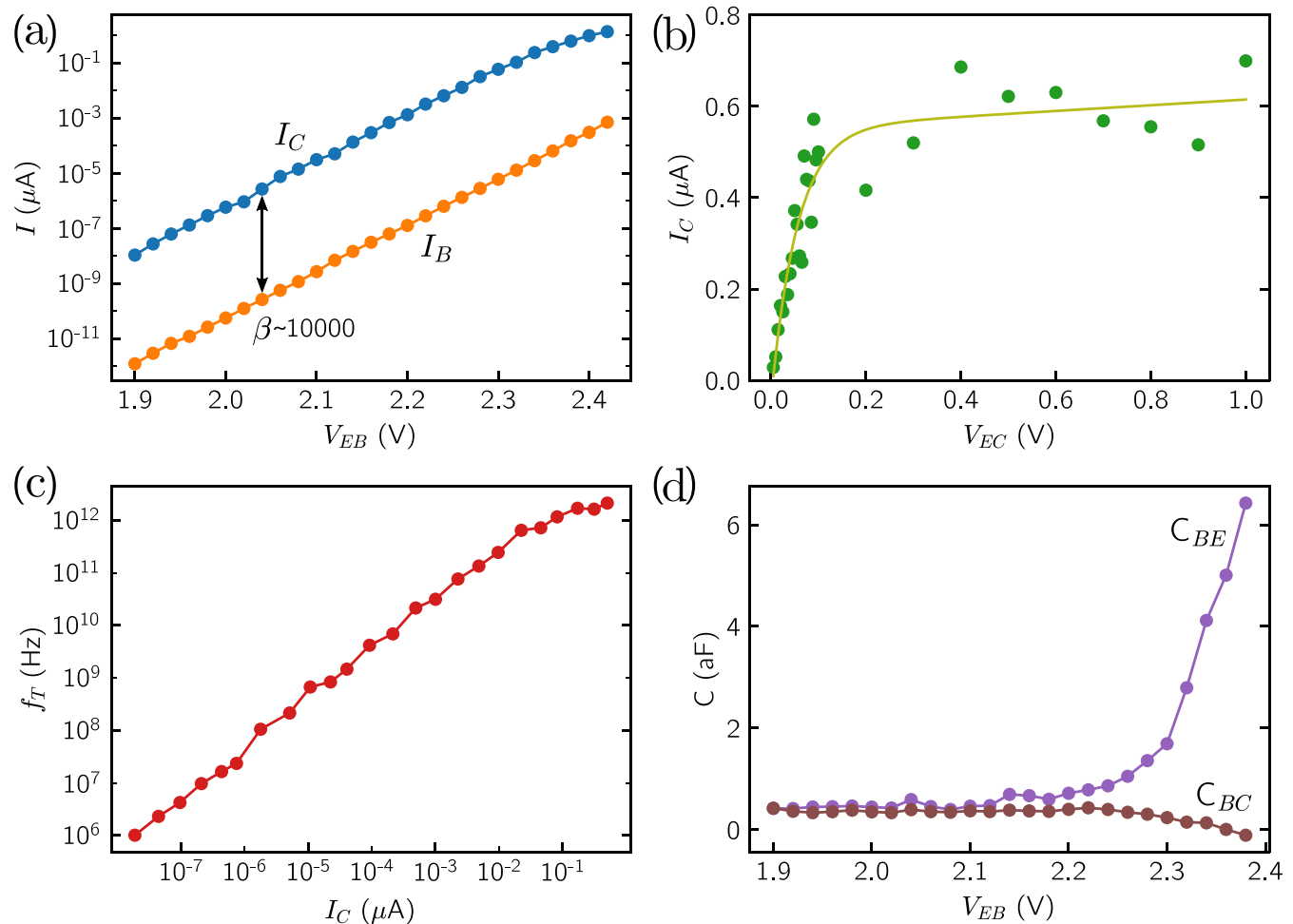

FIG. 3. Performance assessment. (a) $I-V$ plot showing dependence of collector $I_{\mathrm{C}}$ and base $I_{\mathrm{B}}$ currents on $V_{\mathrm{EB}} \equiv q^{-1}\left(\mu_{\mathrm{B}}-\mu_{\mathrm{E}}\right)$ for $V_{\mathrm{EC}} \equiv$ $q^{-1}\left(\mu_{\mathrm{C}}-\mu_{\mathrm{E}}\right)$ fixed to $V_{\mathrm{EC}}=0.5 \mathrm{~V}$. Both currents show exponential behavior with a $\sim 60 \mathrm{mV} /$ decade slope. The almost constant current gain $\beta=I_{\mathrm{C}} / I_{\mathrm{B}}$ is easily inferrable to be $\sim 10000$, dropping to $\sim 6000$ deep in the ON state because of saturation. (b) Dependence of collector current $I_{\mathrm{C}}$ on $V_{\mathrm{EC}}$ for $V_{\mathrm{EB}}=2.38 \mathrm{~V}$ (ON state). The Ebers-Moll model fits our results with a reasonable mismatch. (c) The cutoff frequency $f_{T}$ as a function of the collector current $I_{\mathrm{C}}$. (d) Capacitances' $C_{\mathrm{BE}}$ and $C_{\mathrm{BC}}$ dependence on $V_{\mathrm{EB}}$. Both are almost constant for most of our voltage span.

heterojunctions and their depletion layers. In the bulk, local equilibrium should ensure a vanishing electric field. Otherwise, carriers would flow and neutralize it. This corresponds to a constant electrostatic potential, only obtainable with charge neutrality. The main current components are represented by hole flow from the emitter to collector over the barrier $\left(I_{\mathrm{C}}\right)$, electron flow from the base to emitter $\left(I_{\mathrm{BE}}\right)$ and hole flow from the emitter to base $\left(I_{\mathrm{EB}}\right)$, as shown in Figs. 1(c) and 1(d). The base current $I_{\mathrm{B}}$ is obtained as $I_{\mathrm{B}}=I_{\mathrm{BE}}+I_{\mathrm{EB}}$. In order to keep the current gain $\beta=I_{\mathrm{C}} / I_{\mathrm{B}}$ as high as possible, we need to keep the carrier flow to and from the base as small as possible. While $I_{\mathrm{BE}}$ can be controlled independently via the difference between the emitter conduction band edge and $\mu_{\mathrm{B}}$ (e.g., lowering base doping), $I_{\mathrm{EB}}$ and $I_{\mathrm{C}}$ are both controlled by the difference between the emitter chemical potential $\mu_{\mathrm{E}}$ and the base valence band edge. This issue can be solved by introducing a barrier for holes in the base lead, here obtained by incremental donor doping in the base lead [Fig. 2(c)]. The flat potential profile in the base and the working principle are demonstrated in Fig. 2(a), where we show a section of the local density of states (LDOS) along a path from the emitter to collector for $\mu_{\mathrm{B}}=1.90$ and $2.38 \mathrm{eV}$. As expected, we find that the LDOS away from the heterojunctions corresponds to the bulk LDOS shifted by a flat electrostatic potential. The carrier injection depicted in Figs. 2(b) and 2(c) also confirms the working principle, as we can see that the fixed charge has been effectively neutralized by the injected carriers in all leads, ensuring the charge neutrality condition for both the $\mathrm{ON}$ and the OFF states.

\section{B. Device characteristics}

In Fig. 3(a) we plot the collector current $I_{\mathrm{C}}$ and the base current $I_{\mathrm{B}}$ as a function of $V_{\mathrm{EB}} \equiv q^{-1}\left(\mu_{\mathrm{B}}-\mu_{\mathrm{E}}\right)$. Both curves behave exponentially with a current swing of $\sim 60$ $\mathrm{mV} /$ decade, typical for very well-controlled thermionic currents [20]. In the case of the MOSFET we would have called this quantity "subthreshold swing," but in the case of BJT it would not be appropriate, since the exponential behavior extends for several orders of magnitude up to very high current density. This implies that the electrostatic potential in the base closely follows the electrochemical potential $\Delta \phi_{\mathrm{B}} \simeq \Delta \mu_{\mathrm{B}}$, i.e., as the potential is controlled just by modifying $\mu_{\mathrm{B}}$, i.e., the carrier injection from the base lead. The very steep exponential dependence of $I_{\mathrm{C}}$ on $V_{\mathrm{EB}}$ enables a large current modulation $\left(I_{\mathrm{ON}} / I_{\mathrm{OFF}}\right) \simeq 10^{8}$ for $\Delta V_{\mathrm{EB}}=0.5 \mathrm{~V}$, higher than what is typically achievable with nanoscale MOSFETs. As in common BJTs, increased control comes with the price of a finite base current $I_{\mathrm{B}}$. The current gain $\beta=I_{\mathrm{C}} / I_{\mathrm{B}}$ is highlighted in Fig. 3(a), reaching the value of $\beta \simeq 10^{4}$. For larger $V_{\mathrm{BE}}$, $\beta$ drops to $\sim 6000$. This is due to saturation, as we can also see from the LDOS in Fig. 2(a) with the base barrier being above the emitter chemical potential. This large value for $\beta$ is mainly due to the presence of the barrier for holes in the base lead, shown in Fig. 1(d). Without that barrier, the best $\beta$ 

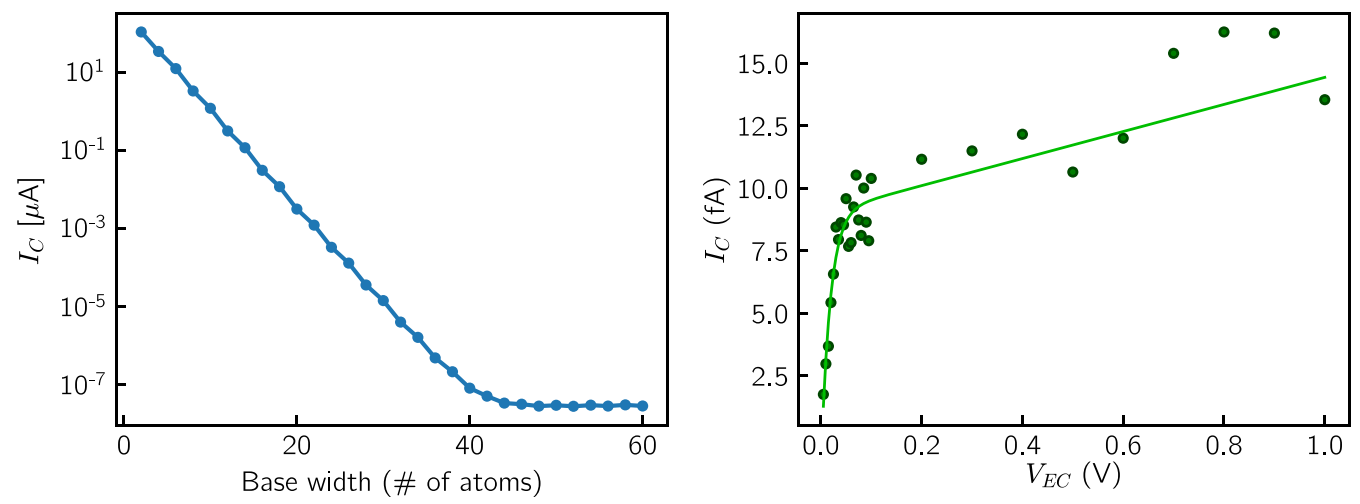

FIG. 4. Left: Collector current $I_{\mathrm{C}}$ dependence on base width for an OFF state channel. Right: Dependence of collector current $I_{\mathrm{C}}$ on $V_{\mathrm{EC}}$ for $V_{\mathrm{EB}}=1.90 \mathrm{~V}$ (OFF state). The Ebers-Moll model fits our results with a reasonable mismatch also in this state.

obtainable would be approximately $\sim 5$, close to what was experimentally observed in Ref. [12]. We implemented this barrier by increasing doping, but in principle it could be created in different ways, e.g., via a Schottky junction or electrostatic doping with a gate electrode. The plot in Fig. 3(b) represents the dependence of the collector current $I_{\mathrm{C}}$ on the emittercollector bias $V_{\mathrm{EC}}$ in the ON state (the OFF state is shown in Fig. 4 and discussed in Appendix E). Here, the noise deriving from the suboptimal convergence is more evident because of the linear scale. Despite the noise, the simulation results can be fitted with an Ebers-Moll model, confirming that our simulation correctly reproduces the essential physics of this device. The cutoff frequency $f_{T}$ is computed quasistatically as

$$
f_{T}=\frac{\frac{\partial I_{\mathrm{C}}}{\partial V_{\mathrm{EB}}}}{2 \pi \frac{\partial Q}{\partial V_{\mathrm{EB}}}},
$$

where $Q$ is the total mobile charge in the device, and is shown in Fig. 3(c), again showing signs of convergence-related noise. Given our assumptions of ballistic transport and high doping in the base, the cutoff frequency steeply increases with the collector current of several orders of magnitude up to the $\mathrm{THz}$ regime, and never reaches the high-injection regime. Both the high $\beta$ and the cutoff frequency results are very promising and are connected to the extremely short base of the BJT, to the high doping, and to the assumption of ballistic transport in the base (see Appendix B). This means that they are optimistic if compared to present-day fabrication capabilities, but they indicate a potential for applications. The differential capacitances $C_{\mathrm{BE}}=\partial Q_{\mathrm{E}} / \partial \mu_{\mathrm{B}}$ and $C_{\mathrm{BC}}=\partial Q_{\mathrm{C}} / \partial \mu_{\mathrm{B}}$, where $Q_{\mathrm{E}}\left(Q_{\mathrm{C}}\right)$ is the total charge injected by $\mathrm{E}(\mathrm{C})$, are plotted in Fig. 3(d) as a function of $V_{\mathrm{EB}}$.

\section{CONCLUSION}

We have proposed and investigated the device concept for the intrinsically thinnest transistor: a nanoscale 2D double heterojunction bipolar transistor using a lateral type-II heterostructure of $\mathrm{WSe}_{2}$ and $\mathrm{MoS}_{2}$. We have shown that this device concept preserves many of the well-known beautiful features of traditional bipolar transistors, such a tenfold increase of the current for an increase of $60 \mathrm{mV}$ of the baseemitter voltage (a "current swing" of $60 \mathrm{mV} /$ decade) over several orders of magnitude of collector current, which is hard to obtain in the case of nanoscale MOSFETs and would require sophisticated multigate and electrostatic engineering. We have also shown that in principle a high current gain of a few thousands is achievable in the ballistic transport assumption if the base lead is properly engineered, even at a low current bias, much higher than obtained in most experiments $[12,16,17]$ and comparable to what has been obtained in the best case [15]. The proposed device is intrinsically promising for high-frequency applications, in terms of cutoff frequency and current gain, and also for high-performance digital applications, when MOSFET leakage currents are comparable with the base current of the BJT. In order for this potential to be actually achieved, several technological improvements have to be achieved, in particular concerning the possibility of doping TMDCs at large molar fractions, and of fabricating high-quality heterojunctions and short base regions.

\section{ACKNOWLEDGMENTS}

The work has been partially supported by the European Commission through the h2020 FET project QUEFORMAL (Contract No. 829035) and by the Ministero dell'Istruzione, dell'Università e della Ricerca through the PRIN project FIVE2D (Contract No. 2017SRYEJH_001).

\section{APPENDIX A: SIMULATION SETUP}

Within our model, any material can be described by three parameters: conduction band edge $E_{\mathrm{CB}}$, valence band edge $E_{\mathrm{VB}}$, and a single effective mass $m^{*}$. Our choice of materials and setup has been inspired by recent work on the creation of lateral heterostructures $[10,11]$. Our device is built to be a $\mathrm{T}$-shaped heterostructure, where the central region is made of $\mathrm{MoS}_{2}$ and the lateral regions are made of $\mathrm{WSe}_{2}$. We used the band alignment from Ref. [21] and the hole effective masses from Ref. [22], setting $E_{\mathrm{gap}, \mathrm{WSe}_{2}}=2.07 \mathrm{eV}$, $E_{\text {gap }, \mathrm{MoS}_{2}}=2.15 \mathrm{eV}, \mathrm{CBO}=0.76 \mathrm{eV}$ (conduction band offset, $\mathrm{MoS}_{2}$ lower), $m_{\mathrm{WSe}_{2}}^{*}=0.46 m_{0}$, and $m_{\mathrm{MoS}_{2}}^{*}=0.64 m_{0}$, as shown in Fig. 1(b). The approximation of taking the hole effective mass for both carriers is reasonable for the materials considered in this work, since for $m_{e, \mathrm{WSe}_{2}}^{*}=$ $0.35 m_{0}$ and $m_{e, \mathrm{MoS}_{2}}^{*}=0.56 m_{0}$. The use of a single effective mass comes from the nearest-neighbor hopping model. A next-to-nearest-neighbor model would allow us to have 
different effective masses for electrons and holes, but it would force us to double the dimension of the subsystems used for the computation (see below), leading to an increase in computational time of $2^{2.7}-6.5$ times. Since the source-drain current is carried by holes, we believe that this approximation does not qualitatively affect our results.

We computed the system Green's function via the recursive Green's function (RGF) algorithm [13,23] implemented in NANOTCAD VIDES. RGF requires the division of the system in parts called "slices" along the transport direction, interacting only between nearest neighbors. Every matrix quantity such as Green's function or self-energies is divided in blocks corresponding to slices. While this procedure is best suited for two leads attached to entire single slices, we implemented the third lead through the Meir-Wingreen formula for transmission [24] and the spectral function

$$
\begin{aligned}
& T_{I, J}=\operatorname{Tr}\left(G_{i j}^{\dagger} \Gamma_{I} G_{i j} \Gamma_{J}\right), \\
& A_{I \alpha}=G_{\alpha i} \Gamma_{I} G_{i \alpha}^{\dagger},
\end{aligned}
$$

where $i, j$ are the device sites belonging to leads $I, J, \alpha$ is a site belonging to the device, $G_{i j}^{(\dagger)}$ are the Green's function blocks corresponding to the sets of atoms $i, j$, and $\Gamma_{I}=$ $i\left(\Sigma_{I}-\Sigma_{I}^{\dagger}\right)$ is the broadening matrix corresponding to the lead self-energy $\Sigma_{I}$. The implementation runs through the broadening matrices, used as projectors on the set of sites belonging to the corresponding lead. If the nonzero parts of the broadening matrices of two leads do not intersect with each other, we may have then two leads attached to the same slice of the device. Further details can be found in Appendix C.

The doping molar fraction is $10^{-1}$ for $n^{+}$and $p^{+}$and $3 \times 10^{-2}$ for $n$, which are higher than experimentally feasible, but required for numerical stability of the computation. Indeed, the fixed charge $\rho_{\text {fixed }}$ makes the solution of the Poisson equation

$$
\nabla \cdot(\epsilon \nabla \phi)=4 \pi\left(\rho_{\text {free }}+\rho_{\text {fixed }}\right)
$$

stable towards variations in the free charge $\rho_{\text {free }}$. We believe this assumption of high doping does not affect the conclusions of this work, as only the presence of doping and the ratios of dopant densities between adjacent regions are important for the device operation described above.

\section{APPENDIX B: DEVICE OPTIMIZATION AND BALLISTIC APPROXIMATION}

We can summarize the device geometry as a T-shaped $\mathrm{MoS}_{2}$ structure, with the horizontal part being a $\sim 10 \times 3-\mathrm{nm}$ armchair nanoribbon and the vertical part being a $\sim 5-\mathrm{nm}$ wide zigzag nanoribbon. Two $\mathrm{WSe}_{2}$ armchair nanoribbons extend the horizontal part, forming two lateral heterostructures. The vertical part forms a curve to have a horizontal end, because the recursive Green's function algorithm requires leads oriented in the transport direction, as self-energies can only connect neighboring slices (see Appendix C). The presence of a curve represents just a numerical complication, as the general device mechanism only requires a generic source injecting carriers in the central region. We consider the shortest possible base that avoids punch-through [20], that in this case is $10 \mathrm{~nm}$ (see Appendix D). This makes it possible for us to assume ballistic transport in the base region, considering that the mean free path in $\mathrm{MoS}_{2}$ is $8 \mathrm{~nm}[5,20]$ and making an approximation that is justified in the context of a device concept investigation, since our results do not critically depend on coherence. In this regime, a large part of carriers ( $\sim 50 \%$ ) would propagate freely through the base, behaving as we described. The other part would undergo phonon scattering events while propagating through the base, mostly losing phase coherence, but retaining most of the initial momentum. Therefore, we believe that a limited amount of scattering would not critically affect our figures of merit, which depend mainly on charge transport, not on phase coherence.

\section{APPENDIX C: THIRD LEAD IMPLEMENTATION}

As mentioned in the main text, we implemented the third lead in our simulations through the broadening matrices $\Gamma_{I}(E)=i\left[\Sigma_{I}(E)-\Sigma_{I}^{\dagger}(E)\right]$, where $\Sigma_{I}(E)$ is the self-energy representing lead $I$. These matrices roughly represent the "spillover" of lead states onto device states. They are present in the formulas for both physical quantities we are interested in, namely the spectral function for states coming from lead $I$, $A_{I}$ [14], and the transmission coefficient from lead $I$ to lead $J$, $T_{I, J}[14,24]$,

$$
\begin{aligned}
& A_{I \alpha}=G_{\alpha i} \Gamma_{I} G_{i \alpha}^{\dagger}, \\
& T_{I, J}=\operatorname{Tr}\left(G_{i j}^{\dagger} \Gamma_{I} G_{i j} \Gamma_{J}\right) .
\end{aligned}
$$

Both equations are written in block matrix form, with $i(j)$ representing the set of device sites connected to lead $I(J)$ by the off-diagonal part of the Hamiltonian, representing hopping processes. This means that $\Gamma_{I}$ has null elements almost everywhere, except on the set of sites connected to lead $I$. Hence, $\Gamma_{I}$ effectively acts as a projector on that set of basis elements. We can use this idea to tweak the recursive Green's function algorithm (RGF) [13], usually employed for two lead simulations, into describing a general number of leads.

RGF requires the division in nearest-neighbor interacting subsystems called slices, implying a block tridiagonal $G^{-1}=(E-H-\Sigma)$. Therefore, we cannot have self-energy connecting sites belonging to different slices. However, this condition does not forbid us to place more than two selfenergies, as long as we place each one on a single slice. Furthermore, we can also have more than one self-energy acting on the same slice, as long as they act on different sites. This is the idea we used in order to introduce more than two leads in the powerful, but limited, RGF framework.

We can make a useful example with an hypothetical system made of three slices each containing four sites. If we had two leads connected to the first and the last slice, we would have

$$
H=\left(\begin{array}{c|c|c}
H_{11} & H_{12} & 0 \\
\hline H_{21} & H_{22} & H_{23} \\
\hline 0 & H_{32} & H_{33}
\end{array}\right), \quad \Sigma=\left(\begin{array}{c|c|c}
\Sigma_{1} & 0 & 0 \\
\hline 0 & 0 & 0 \\
\hline 0 & 0 & \Sigma_{3}
\end{array}\right),
$$

with matrices written in block matrix notation with every block being a $4 \times 4$ matrix. If the two leads are connected to 
the entire first and last slices with self-energies $\sigma, \sigma^{\prime}$, then

$$
\begin{aligned}
& \Sigma_{1}=\left(\begin{array}{llll}
\sigma_{11} & \sigma_{12} & \sigma_{13} & \sigma_{14} \\
\sigma_{21} & \sigma_{22} & \sigma_{23} & \sigma_{24} \\
\sigma_{31} & \sigma_{32} & \sigma_{33} & \sigma_{34} \\
\sigma_{41} & \sigma_{42} & \sigma_{43} & \sigma_{44}
\end{array}\right), \\
& \Sigma_{2}=\left(\begin{array}{llll}
\sigma_{11}^{\prime} & \sigma_{12}^{\prime} & \sigma_{13}^{\prime} & \sigma_{14}^{\prime} \\
\sigma_{21}^{\prime} & \sigma_{22}^{\prime} & \sigma_{23}^{\prime} & \sigma_{24}^{\prime} \\
\sigma_{31}^{\prime} & \sigma_{32}^{\prime} & \sigma_{33}^{\prime} & \sigma_{34}^{\prime} \\
\sigma_{41}^{\prime} & \sigma_{42}^{\prime} & \sigma_{43}^{\prime} & \sigma_{44}^{\prime}
\end{array}\right) .
\end{aligned}
$$

If we want to have two leads each attached to two sites of the first slice instead, we can write the two $2 \times 2$ self-energies $\sigma, \sigma^{\prime \prime}$ and the $4 \times 4$ self-energy $\sigma^{\prime}$ as

$$
\begin{aligned}
\Sigma_{1} & =\left(\begin{array}{cccc}
\sigma_{11} & \sigma_{12} & 0 & 0 \\
\sigma_{21} & \sigma_{22} & 0 & 0 \\
0 & 0 & \sigma_{11}^{\prime \prime} & \sigma_{12}^{\prime \prime} \\
0 & 0 & \sigma_{21}^{\prime \prime} & \sigma_{22}^{\prime \prime}
\end{array}\right) \\
\Sigma_{2} & =\left(\begin{array}{llll}
\sigma_{11}^{\prime} & \sigma_{12}^{\prime} & \sigma_{13}^{\prime} & \sigma_{14}^{\prime} \\
\sigma_{21}^{\prime} & \sigma_{22}^{\prime} & \sigma_{23}^{\prime} & \sigma_{24}^{\prime} \\
\sigma_{31}^{\prime} & \sigma_{32}^{\prime} & \sigma_{33}^{\prime} & \sigma_{34}^{\prime} \\
\sigma_{41}^{\prime} & \sigma_{42}^{\prime} & \sigma_{43}^{\prime} & \sigma_{44}^{\prime}
\end{array}\right) .
\end{aligned}
$$

We can recast the first self-energy block element in two selfenergy block elements,

$$
\sigma=\left(\begin{array}{cccc}
\sigma_{11} & \sigma_{12} & 0 & 0 \\
\sigma_{21} & \sigma_{22} & 0 & 0 \\
0 & 0 & 0 & 0 \\
0 & 0 & 0 & 0
\end{array}\right), \quad \sigma^{\prime \prime}=\left(\begin{array}{cccc}
0 & 0 & 0 & 0 \\
0 & 0 & 0 & 0 \\
0 & 0 & \sigma_{11}^{\prime \prime} & \sigma_{12}^{\prime \prime} \\
0 & 0 & \sigma_{21}^{\prime \prime} & \sigma_{22}^{\prime \prime}
\end{array}\right),
$$

that can be used in order to compute the broadening matrices $\Gamma, \Gamma^{\prime \prime}$. These matrices will have the same block structure and then act as projectors for any physical quantity we want to compute. The former procedure works also for the MeirWingreen formula $T_{I, J}=\operatorname{Tr}\left(G_{i j}^{\dagger} \Gamma_{I} G_{i j} \Gamma_{J}\right)$, where we can now finally say that $i, j$ are sets of atoms corresponding to entire slices. For a proof of this formula, we suggest to the reader the original article [24] and Lake et al. [13].

\section{APPENDIX D: TUNNELING ACROSS BASE AND PUNCH-THROUGH}

Quantum tunneling is a phenomenon that is well represented in the NEGF picture. Therefore, we can have hole states propagating in the base at energies situated in the base material's gap. In our device, we want to avoid a tunneling current. This current depends very weakly on the base chemical potential $\mu_{\mathrm{B}}$, and it introduces a minimum current when we try to switch off the device. This strongly limits the $I_{\mathrm{ON}} / I_{\mathrm{OFF}}$ figure of merit. The effective mass Hamiltonian only gives a qualitatively correct description of quantum tunneling, since the matrix elements merely reproduce the right gaps and effective masses. Nevertheless, we could in principle describe the tunneling probability via the WKB approximation, as we are using a free-particle effective mass model. WKB tunneling predicts the tunneling probability to scale exponentially with the barrier width $P \sim e^{-w / l_{0}}$. This validates what we obtain when we simulate the dependence of the collector current $I_{\mathrm{C}}$ on the base width in the case of a flat barrier constant all over the base, here shown in Fig. 4. For this test, we simulated the channel alone in the OFF state $\left(V_{\mathrm{EB}}=0.0 \mathrm{~V}\right.$, $V_{\mathrm{EC}}=0.5 \mathrm{~V}$ ), assuming perfect charge neutrality in order to write the potential. The current decays exponentially as the base width increases, until it saturates to the OFF state current we presented in the main work. In the plot presented in Fig. 4, we can see that the tunneling current becomes neglectable with respect to the thermionic current for a base larger than 48 atoms, corresponding to $\sim 6 \mathrm{~nm}$. The base width was finally chosen to be $\sim 10 \mathrm{~nm}$ wide because of the presence of $\sim 1.5$-nm-wide depletion layers spanning from both heterojunctions.

\section{APPENDIX E: COLLECTOR CURRENT DEPENDENCE ON SUPPLY VOLTAGE IN THE OFF STATE}

We show here in Fig. 4 the dependence of the collector current $I_{\mathrm{C}}$ on the supply voltage $V_{\mathrm{EC}}$ for $V_{\mathrm{EB}}=1.90 \mathrm{~V}$, corresponding to the OFF state. This plot helps us to assess the behavior of our device. Since the data obtained from the simulations can be fitted with the Ebers-Moll model equation, we can confirm that our device works as a BJT in both the OFF and the ON states.
[1] W. Shockley, Circuit element utilizing semiconductive material, U. S. Patent 2,569,347 (25 September 1951).

[2] W. Quan, A. M. Arabhavi, R. Flückiger, O. Ostinelli, and C. R. Bolognesi, Quaternary graded-base InP/GaInAsSb DHBTs with $f_{\mathrm{T}} / f_{\mathrm{MAX}}=547 / 784 \mathrm{GHz}$, IEEE Electron Device Lett. 39, 1141 (2018).

[3] G. Fiori, F. Bonaccorso, G. Iannaccone, T. Palacios, D. Neumaier, A. Seabaugh, S. K. Banerjee, and L. Colombo, Electronics based on two-dimensional materials, Nat. Nanotechnol. 9, 768 (2014).

[4] G. Iannaccone, F. Bonaccorso, L. Colombo, and G. Fiori, Quantum engineering of transistors based on 2D materials heterostructures, Nat. Nanotechnol. 13, 183 (2018).
[5] B. Radisavljevic, A. Radenovic, J. Brivio, V. Giacometti, and A. Kis, Single-layer $\mathrm{MoS}_{2}$ transistors, Nat. Nanotechnol. 6, 147 (2011).

[6] H. Fang, S. Chuang, T. C. Chang, K. Takei, T. Takahashi, and A. Javey, High-performance single layered $\mathrm{WSe}_{2}$ p-FETs with chemically doped contacts, Nano Lett. 12, 3788 (2012).

[7] D. Jariwala, V. K. Sangwan, L. J. Lauhon, T. J. Marks, and M. C. Hersam, Emerging device applications for semiconducting twodimensional transition metal dichalcogenides, ACS Nano 8, 1102 (2014).

[8] S. Manzeli, D. Ovchinnikov, D. Pasquier, O. V. Yazyev, and A. Kis, 2D transition metal dichalcogenides, Nat. Rev. Mater. 2, 17033 (2017). 
[9] Z. Cai, B. Liu, X. Zou, and H.-M. Cheng, Chemical vapor deposition growth and applications of two-dimensional materials and their heterostructures, Chem. Rev. 118, 6091 (2018).

[10] M.-Y. Li, Y. Shi, C.-C. Cheng, L.-S. Lu, Y.-C. Lin, H.-L. Tang, M.-L. Tsai, C.-W. Chu, K.-H. Wei, J.-H. He, W.-H. Chang, K. Suenaga, and L.-J. Li, Epitaxial growth of a monolayer $\mathrm{WSe}_{2}-\mathrm{MoS}_{2}$ lateral p-n junction with an atomically sharp interface, Science 349, 524 (2015).

[11] Y. Han, M.-Y. Li, G.-S. Jung, M. A. Marsalis, Z. Qin, M. J. Buehler, L.-J. Li, and D. A. Muller, Sub-nanometre channels embedded in two-dimensional materials, Nat. Mater. 17, 129 (2017).

[12] C.-Y. Lin, X. Zhu, S.-H. Tsai, S.-P. Tsai, S. Lei, Y. Shi, L.-J. Li, S.-J. Huang, W.-F. Wu, W.-K. Yeh, Y.-K. Su, K. L. Wang, and Y.-W. Lan, Atomic-monolayer two-dimensional lateral quasi-heterojunction bipolar transistors with resonant tunneling phenomenon, ACS Nano 11, 11015 (2017).

[13] R. Lake, G. Klimeck, R. Chris Bowen, and D. Jovanovic, Single and multiband modeling of quantum electron transport through layered semiconductor devices, J. Appl. Phys. 81, 7845 (1997).

[14] S. Datta, Quantum Transport: Atom to Transistor (Cambridge University Press, Cambridge, UK, 2005).

[15] P. Agnihotri, P. Dhakras, and J. U. Lee, Bipolar junction transistors in two-dimensional $\mathrm{WSe}_{2}$ with large current and photocurrent gains, Nano Lett. 16, 4355 (2016).

[16] L. Liu, N. Xu, Y. Zhang, P. Zhao, H. Chen, and S. Deng, van der Waals bipolar junction transistor using vertically stacked two- dimensional atomic crystals, Adv. Funct. Mater. 29, 1807893 (2019).

[17] B.-W. Su, B.-W. Yao, X.-L. Zhang, K.-X. Huang, D.-K. Li, H.-W. Guo, X.-K. Li, X.-D. Chen, Z.-B. Liu, and J.-G. Tian, A gate-tunable symmetric bipolar junction transistor fabricated via femtosecond laser processing, Nanoscale Adv. 2, 1733 (2020).

[18] NanoTCAD ViDES website, http://vides.nanotcad.com/vides/.

[19] T. K. Agarwal, B. Soree, I. Radu, P. Raghavan, G. Iannaccone, G. Fiori, W. Dehaene, and M. Heyns, Material-device-circuit co-optimization of 2D material based FETs for ultra-scaled technology nodes, Sci. Rep. 7, 5016 (2017).

[20] S. M. Sze and K. K. Ng, Physics of Semiconductor Devices (Wiley, Hoboken, NJ, 2006).

[21] M.-H. Chiu, C. Zhang, H.-W. Shiu, C.-P. Chuu, C.-H. Chen, C.-Y. S. Chang, C.-H. Chen, M.-Y. Chou, C.-K. Shih, and L.-J. Li, Determination of band alignment in the single-layer $\mathrm{MoS}_{2} / \mathrm{WSe}_{2}$ heterojunction, Nat. Commun. 6, 7666 (2015).

[22] J. Chang, L. F. Register, and S. K. Banerjee, Ballistic performance comparison of monolayer transition metal dichalcogenide $M_{2}(M=\mathrm{Mo}, \mathrm{W} ; X=\mathrm{S}, \mathrm{Se}, \mathrm{Te})$ metaloxide-semiconductor field effect transistors, J. Appl. Phys. 115, 084506 (2014).

[23] D. J. Thouless and S. Kirkpatrick, Conductivity of the disordered linear chain, J. Phys. C 14, 235 (1981).

[24] Y. Meir and N. S. Wingreen, Landauer Formula for the Current through an Interacting Electron Region, Phys. Rev. Lett. 68, 2512 (1992). 